\title{
NON-ERGODIC TO ERGODIC REGIME TRANSITION VIA INTRUSIONS IN A NON-POISSON RENEWAL SYSTEM WITH POWER LAW TAILS
}

\author{
Osman Çağlar Akın \\ Department of Physics, Fatih University, 34500 \\ Büyükçekmece, İstanbul, Turkey \\ ocakin@fatih.edu.tr
}

\begin{abstract}
We study the effect of non-linear perturbations in the form of periodic intrusions in the case of a non-Poisson renewal system in the non-ergodic regime. We notice that, intrusions may cause the system to shift the power index and may even cause a transition from a non-ergodic regime to an ergodic regime. We have made the diffusion entropy analysis (DEA) of the system and notice that the change of the degree of complexity is consistent with the transition as well.
\end{abstract}

Key Words- Stochastic processes, renewal theory

\section{INTRODUCTION}

Probability distribution functions with power law tails have been observed in many natural systems. Among them are some systems like the dynamics of human brain [1] and the on-off processes of blinking quantum dots [2], [3] which have already been shown to be obeying a non-Poisson renewal process waiting time distribution function prescription.

The non-Poisson renewal systems with long power law tails may be classified in three groups. The first group is the one in which we have the first moment and the second moment of the probability distribution function to be finite, which occurs when the power index $\mu>3$, this regime is a non-Poisson system in the ergodic regime but with a finite second moment. The second group is for the case where $2<\mu<3$, those distributions with a finite expectation value but an infinite second moment. In this case we may define a finite value for the average value of the independent variable $\tau$ and the system is referred to as a non-Poisson renewal system in the ergodic regime with an unbounded second moment. The third case occurs when the power index obeys $1<\mu<2$, where the probability distribution function itself can be normalized, yet neither the average value, nor the second moment of the distribution is finite. This case is observed in nature in the on-off processes of blinking quantum dots and the human brain activity [1], [2], [3] and is referred to as a non-Poisson renewal process in the nonergodic regime.

In this work we study the effects of intrusive non-linear perturbations on the non-Poison renewal system event production rate in the case of the non-ergodic regime. Previous works in this concern have covered the cases for the effects of Poisson and non-Poisson rate perturbations in the harmonic (or as is referred to by the authors as the linear), and exponential, (or the non-linear) perturbations in the references [4] and [5] respectively. But the investigation of the effects of perturbation in the ergodic region where a finite first moment fails to exist for the probability distribution under intrusive perturbations have been missing. Interestingly enough, this region is also the region 
where we observe waiting time distributions of EEG of the human brain activity data [1] and the blinking quantum dots on-off processes to reside [2], [3].

\subsection{The Poisson Renewal Model}

\section{MODEL}

Here we give a brief derivation of the Poisson Renewal process from the basic principles. In the next subsection we will apply the same principles to derive a nonPoisson Renewal process model. This model is the same model the authors of [4] have utilized. We will use the derivation of the Poisson model to follow as a paradigmatic model to construct and analyze the non-Poisson case as well.

Assume that a Uniform random number $\xi$ is generated in the range $[0,1]$ with zero correlations, ensuring that there will be no memory in the process to be explained below. Based on a comparison with a number $r_{i}<<1$, if the generated uniform random number $\xi<\mathrm{r}_{\mathrm{i}}$ holds, we will record an event as 1 and else no event as 0 , to form a sequence of events and failures which are represented by a sequence of 1's and 0's. Our aim is to obtain an inter-event time interval sequence and eventually its survival probability and probability distribution functions based on this simple model. Note that $\mathrm{r}_{\mathrm{i}}$ may assume different values at each consecutive trial and hence $i$ stands to denote the number of the trial. The probability that there will be no event at the $i^{\text {th }}$ run is given as $\mathrm{p}_{\mathrm{i}}=1-\mathrm{r}_{\mathrm{i}}$. The survival probability that there will be no events after $\mathrm{n}$ trials, or the probability to have $\mathrm{n}$ consecutive failures is

$$
\begin{aligned}
& \Psi(n)=\prod_{i=1}^{n} p_{i}=\prod_{i=1}^{n}\left(1-r_{i}\right) \\
& \ln (\Psi(n))=\ln \left(\prod_{i=1}^{n}\left(1-r_{i}\right)\right)=\sum_{i=1}^{n} \ln \left(1-r_{i}\right)
\end{aligned}
$$

Note that $\ln (1+\mathrm{x}) \approx \mathrm{x} \quad$ for $\mathrm{x}<<1$

$$
\ln (\Psi(n))=-\sum_{i=1}^{n} r_{i}
$$

For the continuous time case this reads an integral

$$
\ln (\Psi(t))=-\int_{0}^{t} r\left(t^{\prime}\right) d t^{\prime}
$$

If we set the rate to be time independent and to be a constant $r_{o}$ then the survival probability will be

$$
\Psi(t)=\exp \left[-r_{o} t\right]
$$

Using the arguments of Renewal theory, the relationship between the probability distribution function and the survival probability is given as [6]

$$
\begin{aligned}
& \psi(t)=-\frac{d \Psi(t)}{d t} \\
& \frac{\psi(t)}{\Psi(t)}=r(t)
\end{aligned}
$$

which implies the well known Poisson Renewal probability distribution function

$$
\psi(\tau)=r_{o} \exp \left(-r_{o} \tau\right)
$$


where $\tau$ stands for the waiting times between successive events which may also be referred to as inter-event time intervals.

\subsection{The non-Poisson renewal model}

The system we derive to obtain the waiting times is based on the same map Paul Manneville [7] used to obtain the durations of the laminar flows in an intermittent flow as

$\mathrm{x}_{\mathrm{n}+1}=\mathrm{x}_{\mathrm{n}}+\mathrm{x}_{\mathrm{n}}{ }^{\mathrm{z}}$

where $\mathrm{x}$ is in the range $[0,1]$ and $\mathrm{z} \geq 1$.

One can prove that a probability distribution functions with power law tails can obtained with $\mu=\mathrm{z} /(\mathrm{z}-1)$, for $\mathrm{z}>1$, and in the limit as $\mathrm{z}$ tends to 1 and hence $\mu$ tends to infinity, to yield a Poisson distribution. So in a single map all the distributions that can be normalized, non-Poisson or Poisson renewal type, can be realized via tuning of a single parameter.

Here we outline a simple model [5] which follows from the Poisson model to make it possible for us to apply our perturbations on the rate in the non-Poisson model. We will apply the same procedure we have used for the Poisson case for a particular time dependent rate function of the form

$$
r\left(t_{k}\right)=\frac{r_{o}}{1+r_{1} \cdot t_{k}}
$$

where where $t_{k}$ is the running time parameter starting from zero, increasing by one every time when there is a failure and will be reset to zero whenever there is an event, henceforth we obtain an event sequence. If we generate the event sequence of failures and events using this time dependent rate,

$$
\begin{aligned}
& \ln (\Psi(t))=-r_{0} \int_{0}^{t} \frac{1}{1+r_{1} \cdot t^{\prime}} d t^{\prime} \\
& \Psi(t)=\left(1+r_{1} \cdot t\right)^{-\left(\frac{r_{o}}{r_{1}}\right)}
\end{aligned}
$$

which then requires the probability distribution function of the inter-event time intervals $\psi(t)=r_{o} \cdot\left(1+r_{1} \cdot t\right)^{-\left(\frac{r_{0}}{r_{1}}+1\right)}$

At this point if for the two parameters we make the following choice with two different variables as

$$
\begin{aligned}
& r_{1}=\frac{1}{T} \\
& r_{o}=\frac{(\mu-1)}{T}
\end{aligned}
$$

The probability distribution function then turns out to be

$$
\psi(\tau)=(\mu-1) \cdot \frac{T^{(\mu-1)}}{(T+\tau)^{\mu}}
$$

where $\tau$ stands for the inter-event time intervals. Here $\mu$ denotes the power index of the renewal system and $\mathrm{T}$ denotes a measure of the transition time to this power law. 
Obviously this transition time is necessary because a power law of the form $\tau^{-\mu}$ can not be sustained with arbitrarily small $\tau$ because $\psi$ blows up to infinity as $\tau$ tends to zero. In this scheme the transition time to power law $\mathrm{T}$ and the power law index $\mu$ are independent parameters and can be perturbed one at a time or both at the same time, as they may appear as connected to physical parameters of the system, internal or external.

\subsection{The non-ergodic regime of the non-Poisson renewal system with power law tails}

We should consider what the value of the power index $\mu$ may inform us about the properties of the waiting time distributions. To have a normalized distribution function

$\int_{0}^{\infty} \psi(\tau) d \tau=1$

and for this condition to be satisfied it is easy to show that $\mu>1$ must be satisfied.

As to the first moment of the waiting time distributions

$<\tau>=\int_{0}^{\infty} \psi(\tau) \cdot \tau \cdot d \tau$

with the power law p.d.f. this requires that the power law index $\mu$ must be larger than 2 to have a finite value for the expectation value of the waiting times.

At this point we must clarify that since the rules generating the statistical distribution does not change in time which implies that the system is stationary, yet the infiniteness of the expectation value of the waiting times with a normalized p.d.f. requires that larger and larger values of the waiting times are likely to be produced in time, and yet the statistics of the system remains the same. Although the system is nonergodic from the point of view of statistics it is stationary [8]. If one follows the above mentioned algorithm to generate the waiting times of the system, before generating a predetermined finite number of waiting times it is probable that the program halts, or else, one has to wait for an indefinite time for the next value of the waiting time to be spelled by the step-wise algorithm, because the expectation value of the waiting times is not finite and in this regime the system is referred to as a non-ergodic renewal system. Such systems are not just mathematical objects and have been observed experimentally in nature [9].

As with the power law index $2<\mu<3$ the system has finite first moment but infinite second moment. This is a power law renewal system in the ergodic regime. As for the case where $3<\mu$, the system has a finite second moment too. As for $\mu \rightarrow \infty$, it can easily be shown that the system tends to a Poisson distribution.

\subsection{The Halting problem}

The non-ergodicity of the non-Poisson renewal system with the above mentioned algorithm implies that the algorithm may halt at some point, or in other words, it may take an indefinitely long time before the system spells out the next waiting time at some point. To obtain a finite number of waiting times with a certain $1<\mu<2$ might take an infinitely long time if the above mentioned algorithm is applied. So for the case where there is no perturbation there is the risk of halting for the 
algorithm. For obvious reasons to be mentioned later in this paper, we do not experience the halting problem for the case of a non-ergodic Renewal system under periodic intrusions. Actually it is possible to circumvent the Halting problem when there are no intrusions or perturbations in the non-ergodic case with yet another algorithm but that is out of the scope of the current paper [10].

\section{INTRUSIVE PERTURBATIONS}

\subsection{Intrusive perturbation as a non-linear perturbation}

Intrusive perturbations on the rate expression may be affecting the power index or the transition time to power law of a system. We will refer to intrusive perturbations here as instant changes in the rate, due to some internal or external parameter of the system, like intensity in the case of a pulsed laser in the presence of some other light source, or an instant pulse of pressure, which may have same effect on the stochastic system parameters. This kind of intrusions do not have to be periodic, they might themselves follow a Poisson distribution with a finite average as in the case of photon arrivals in the human eye, but here for the sake of simplicity of the analysis, we assume a series of intrusions with a perfect periodicity.

\subsection{Intrusions on the Poisson renewal system}

Let us assume the simplest case of periodic intrusions on a Poisson renewal system which may be formulated as follows

$$
r(t)=r_{O}\left(1+A \cdot \delta\left(t-n \cdot T_{\omega}\right)\right)
$$

The event rate makes sudden and instant jumps of strength $A$ with an intrusion period of $\mathrm{T}_{\omega}$.

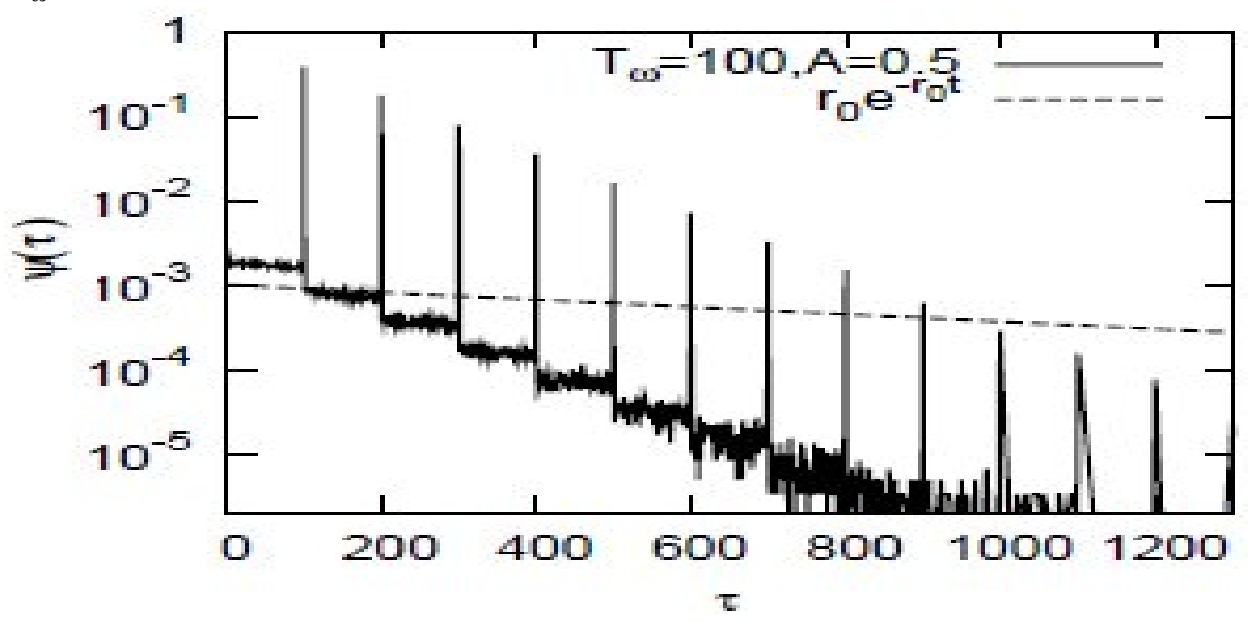

Figure.1. Waiting time distribution with periodic intrusions on the Poisson system $\mathrm{A}=0.5, \mathrm{~T}_{\omega}=100$.

The figure shows that the effective rate of the probability distribution function of the waiting times are modified significantly by the intrusions, though the general behaviour of the distribution is still of the Poisson type. 


\subsection{Intrusions on the non-Poisson renewal system}

For an intrusion on the event production rate parameter $r_{o}$ in the case of nonPoisson renewal process with power law tails, we study the following time dependent rate

$$
r(t)=\frac{r_{o} \cdot\left(1+A \cdot \delta\left(t-n \cdot T_{\omega}\right)\right)}{1+r_{1} \cdot \Delta t}
$$

Where $\Delta \mathrm{t}=\mathrm{t}-\mathrm{t}_{\mathrm{i}-1}$ starts from zero, $\mathrm{t}_{\mathrm{i}-1}$ referring to the time of the former event, $\Delta \mathrm{t}$ being increased by one at every step and reset to zero whenever there is an event, whereas $t$ increases by one at every step and in never reset since it denotes the absolute time. In this scheme A may be referred to as the intrusion strength, and $T_{\omega}$ is the intrusion period. We consider the $\delta$-function to describe a comb function of magnitude one at the positive integer $n$ multiples of the intrusion strength. So basically what this rate describes is an intrusive relative increase for the rate, an intrusive perturbation on $r_{o}$ with a period of $\mathrm{T}_{\omega}$ and a strength of $\mathrm{A}$. We may obtain an expression for the survival probability by utilizing this expression in the integral expression of Equation (4) to get

$$
\ln (\Psi(t))=\ln \left(1+r_{1} \cdot t\right)^{-\frac{r_{o}}{r_{1}}}-r_{o} \cdot A \cdot \sum_{n=1}^{N} \frac{1}{1+r_{1} \cdot n \cdot T_{\omega}}
$$

Here we show the integer value

$$
N=\left[\frac{t}{T_{\omega}}\right]
$$

If we set $r_{1} \mathrm{~T}_{\omega}=\mathrm{a}$, then the second term on the r.h.s. may be written as a function of two digamma functions

$$
\ln (\Psi(t))=\ln \left(1+r_{1} \cdot t\right)^{-\frac{r_{o}}{r_{1}}}-r_{o} \cdot A \cdot \frac{\Phi(N+3)-\Phi(3)}{a}
$$

Here $\Phi$ denotes the digamma function. One can easily notice the behavioural similarity of the digamma function to $l n$-function and hence can define a ratio $\mathrm{C}(\mathrm{N})$

$$
C(N)=\frac{\Phi(N+3)-\Phi(3)}{a \cdot \ln (N)}
$$

And notice that $\mathrm{C}(\mathrm{N})$ is a very slowly changing function of $\mathrm{N}$ which is almost constant over a very wide range, therefore

$$
\begin{aligned}
& \ln (\Psi(t))=\ln \left(1+r_{1} \cdot t\right)^{-\frac{r_{o}}{r_{1}}}+\ln (N)^{-r_{o} \cdot A \cdot \frac{\Phi(N+3)-\Phi(3)}{a \cdot \ln (N)}} \\
& \Psi(t)=\left(1+r_{1} \cdot t\right)^{-\frac{r_{o}}{r_{1}}} \cdot\left[\frac{t}{T_{\omega}}\right]^{\left\{-\frac{r_{o}}{r_{1}} \cdot \frac{A}{T_{\omega}} \cdot \frac{\Phi\left(\left[\frac{t}{T_{\omega}}\right]+3\right)-\Phi(3)}{\left.\ln \left[\frac{t}{T_{\omega}}\right]\right)}\right\}}
\end{aligned}
$$

which means that the survival probability function is multiplied by a slowly varying factor which is modified significantly with a time span of, $\mathrm{T}_{\omega}$. This dependence on the intrusion period is reflected in the simulations for the survival probability function in Figure.2. 


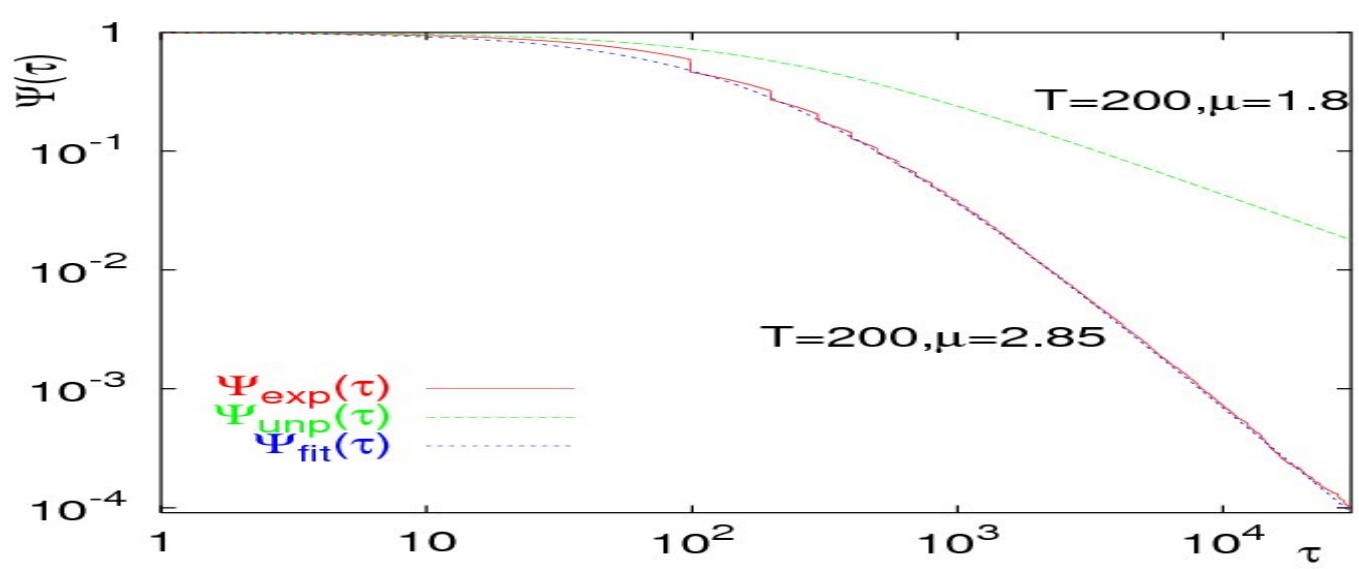

Figure.2. The unperturbed survival probability function for the unperturbed case $\Psi_{\text {unp }}(\tau)$ with $\mu=1.8, \mathrm{~T}=200$, the experimental survival probability distribution $\Psi_{\exp }(\tau)$ under intrusion $\mathrm{A}=0.5, \mathrm{~T}_{\omega}=100$, with $\mu=1.8, \mathrm{~T}=200$ and its fit $\Psi_{\text {fit }}(\tau)$ with $\mu=2.85, \mathrm{~T}=200$.

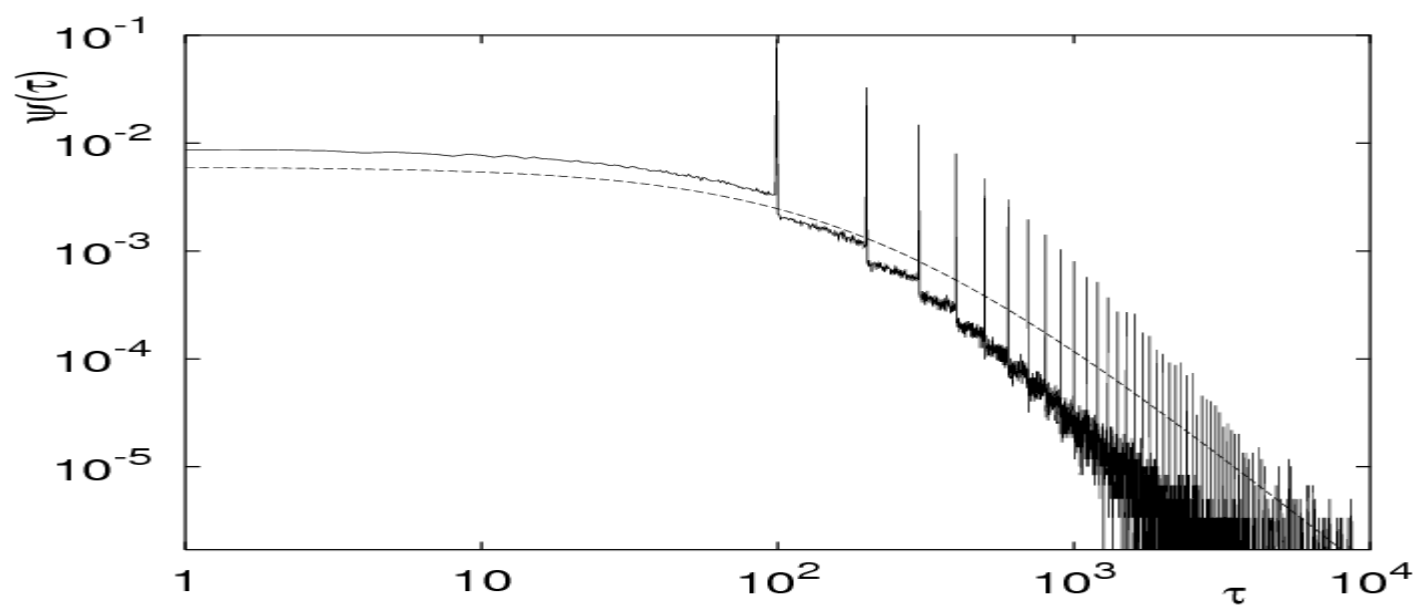

Figure.3. Waiting time distribution for no intrusion $\mu=1.8, \mathrm{~T}=200$ (dashed) and with intrusion $\mathrm{A}=0.5, \mathrm{~T}_{\omega}=100$ (solid line).

There will be a corresponding change in the probability distribution function and hence a different power index may be expected. At this point, a natural question to ask is whether or not in the long run, this expected change in the power index may be reflected in the complexity degree measurement or not.

\section{DIFUSION ENTROPY ANAYSIS AS A MEASURE OF THE DEGREE OF COMPLEXITY OF A SYSTEM}

By the above mentioned algorithm we obtain an event sequence denoted by 1's between a sequence of failures denoted by 0 's. We can obtain the degree of complexity of such a sequence by means of diffusion entropy using asymmetric jump model technique, which essentially gives us the rate of increase of the Shannon entropy (or else otherwise known as the Kolmogorov-Sinai entropy) as the sequence length tends to infinity. By running the diffusion entropy analysis directly on the event sequence obtained from the above mentioned rate with periodic intrusions, we can check whether or not the intrusions also have an effect on the degree of complexity of the system. 
For this reason we give a brief overview of the Diffusion Entropy Analysis [11] which also has a connection to the concept of algorithmic complexity [12]. This techniques relies on converting the time series $\left\{\chi_{i}\right\}$ into a diffusion process.

In principle a Gibbs ensemble of random walks may be produced from the time series to obtain the locations of the random walker after following k steps as $x(t)=\sum_{i=1}^{t} \chi_{i}$

Although we have only one time series, relying on the fact that the system is stationary (which does not necessarily imply ergodicity), we consider mobile windows of size $k$ namely

$x(t, m)=\sum_{i=m}^{m+t} \chi_{i}$

To obtain the position of the $m^{\text {th }}$ random walker. We can convert the original time series to a distribution of locations considering all possible $m$ 's and hence we may obtain a probability distribution function for the positions as a function of time window length. But the probability distribution function of these positions $\mathrm{p}(\mathrm{x}, \mathrm{t})$ might as well be evolving as a function of the window length and in particular if the probability distribution function, regardless of its particular form satisfies the following form

$$
p(x, t)=\frac{1}{t^{\delta}} F\left(\frac{x}{t^{\delta}}\right)
$$

Then it is said that the probability distribution function has scaling property and that the scaling coefficient is $\delta$. Note that the particular form of the function $F$ is irrelevant for the scaling property to exist.

Once we have the probability distribution function as a function of the window length then we can apply the Shannon entropy

$$
S=-\int_{-\infty}^{\infty} p(x, t) \cdot \ln (p(x, t)) \cdot d x
$$

Since the time is discrete

$$
S(t)=-\sum_{i=i}^{K-t+1} p\left(x_{i}, t\right) \cdot \ln \left(p\left(x_{i}, t\right)\right)
$$

where $K$ denotes the total length of the sequence $\left\{\chi_{i}\right\}$, so the summation is over all possible random walker paths.

It is easy to show that provided $\mathrm{p}(\mathrm{x}, \mathrm{t})$ satisfies the scaling condition, the Shannon entropy as a function of the time window behaves as

$S(t)=\delta \cdot \ln (t)+R$

Where $\mathrm{R}$ is a constant determined by the distribution function and is irrelevant at this point. In the $\mathrm{S}(\mathrm{t})$ versus semi-log-t plot of the Shannon entropy obtained from the sequence as a function of the window size, the slope $\delta$, if there is a definite one, will give us the scaling coefficient in case it exists.

Diffusion Entropy Analysis on the Asymmetric Jump Model, i.e., on the event sequence we have obtained, is known to yield the following scaling relations [13] if a scaling relation persists 


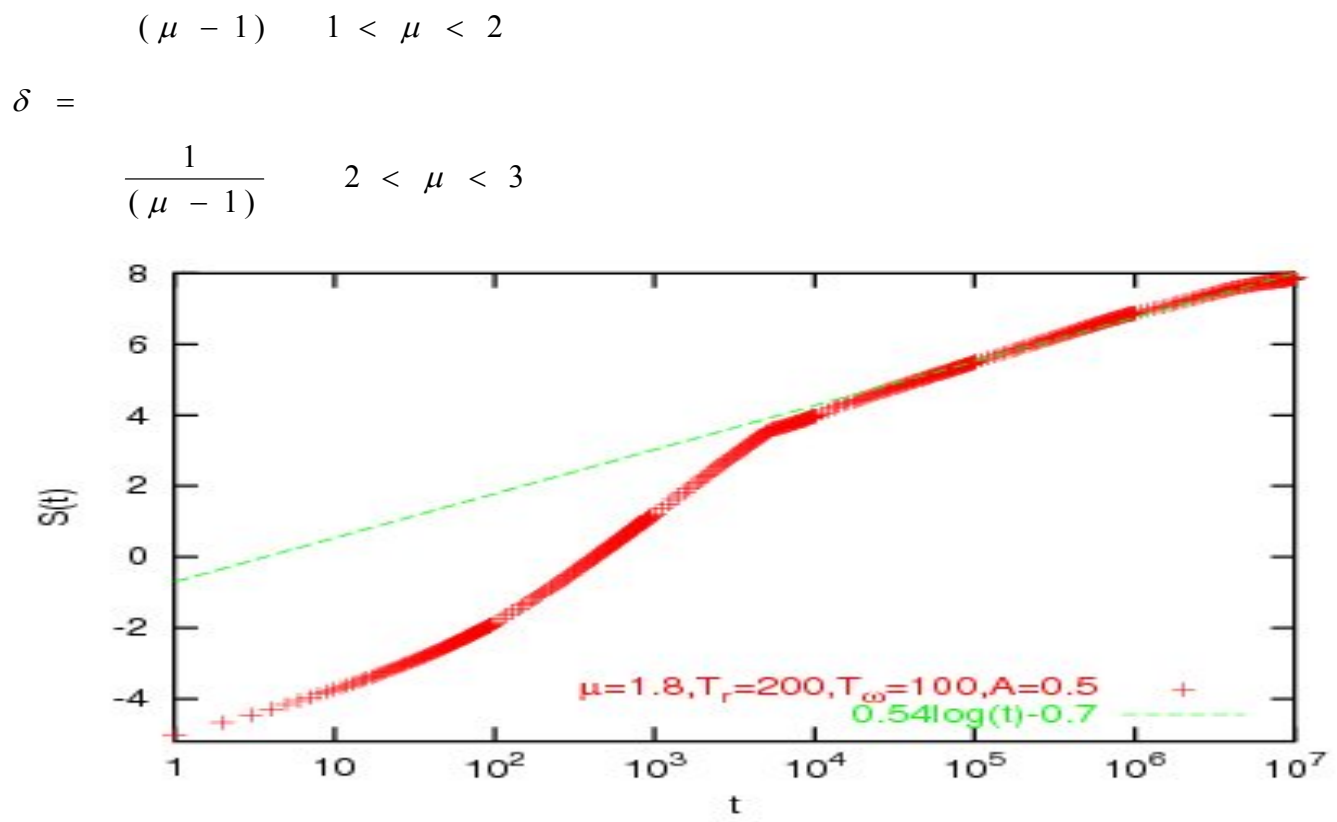

Figure.4. The Diffusion Entropy Analysis on the event sequence under intrusions with $\mathrm{A}=0.5$ and $\mathrm{T}_{\omega}=100$.

The diffusion entropy scaling which is expected for the unperturbed case is $\delta=0.8$ for an unperturbed $\mu=1.8, \mathrm{~T}=200$. For the case of intrusion with $\mathrm{A}=0.5$ and $\mathrm{T}_{\omega}=100$, in the Figure, one can see that, not only the long term power law index $\mu$ is modified under intrusion, but also the corresponding scaling coefficient for the perturbed power index $\mu=2.85$ using Equation (33) is also observed in the Diffusion Entropy Analysis.

\section{CONCLUSIONS}

The non-linear perturbations on the event production rate for the Renewal processes have been studied before in the context of the neuron spike firing inter event time intervals. In this study, we have studied a non-linear perturbation in the form of periodic intrusions on the event production rate. This intrusion may have been caused by an external physical variable like the intensity of a pulsed laser or an internal variable of the system itself. In this study we have shown that the apparent scaling and power law index of the system may have been drastically modified, even so as to make a transition from an originally non-ergodic regime to an ergodic regime, provided the intrusions have an affect on the event production rate $r(t)$ through the parameter $r_{o}$. The case where the intrusions interact with the parameter $r_{1}$ such that the transition time to power law is affected needs special attention and requires further detailed investigations since the entire system then may prove to be non-ergodic probably even after the intrusions are performed. We can predict that, under special circumstances a transition from a non-ergodic to ergodic regime is possible, and that the detection of the power index must be done very carefully in data analysis, since the original system may actually have a different power index then the long time limit detected. Here we have considered a periodic relative increase for the event production rate. It is easy to consider the reverse case where there is a relative decrease in the event production rate periodically, in which case one may expect a decrease of the power index and a 
corresponding change of the scaling. Possible periodic intrusions on the stochastic system may be more visible in the survival probability plots in the form of sudden drops as in the case of Figure.4., hence one must be very careful before concluding about the power index of the system because even Diffusion Entropy Analysis gives consistent results with the modified power index rather than the original one. Such intrusions may be persistent in systems like complex biological systems like heart-brain interactions and the intrusions need not be exactly periodic. For further study, the case of intrusions on the transition time to power law needs detailed digression.

\section{REFERENCES}

1. Bianco S., Ignaccolo M.. Rider M.S. et.al, Brain music and non Poisson renewal processes, Physical Review E: statistical, nonlinear, and softmatter physics, 75(6), 061911, part 1, 2007.

2. Paradisi P. Allegrini P, Barbi F. et.al, Fluorescence intermittency in blinking quantum dots, Journal of Chemical Physics, 123(17), 174704., 2005.

3. P.Paradisi, S.Bianco, P.Grigolini, O.C.Akin, Renewal aging in non-Poisson renewal systems with periodic rate modulation, International Journal of Bifurcation and Chaos, 18(9), 2681-2691, 2008 .

4. Akin O.C., Paradisi P. Grigolini P., Nov 15 2006, Periodic trend and fluctuations: the case of strong correlation, Physica A, Statistical Mechanics and its applications, 371(2), 157-170, 2006.

5. Akin O.C., Paradisi P., Grigolini P., Perturbation induced emergence of Poisson-like behavior in the non-Poisson systems, Journal of Statistical mechanics: Theory and Experiment, P01013, 2009.

6. D.R.Cox., Renewal Theory, Methuen \& Co. Ltd Science paperbacks, London, 1967.

7. P.Manneville., Intermittency, self-similarity and $1 / \mathrm{f}$ spectrum in dissipative dynamical systems, Journal de Physique. 41(11), 1235, 1980.

8. L.S.Liebovitch., The fractal random telegraph signal: signal analysis and applications., Annals of Biomedical Engineering, 16, 483-494, 1988.

9. Nirmal M.., Dabbousi B.O., Bawendi M.G., Macklin J.J., Trautman J.K., Harris T.D., Brus L.E., Fluorescence intermittency in single cadmium selenide nanocrystals, Nature, 383, 802, 1996.

10. Kalashyan A.K., Buiatti M., Grigolini P., Ergodicity breakdown and scaling from single sequences, Chaos Solitons and Fractals, 39, 895-909, 2009.

11. Scafetta N., Grigolini P., Scaling detection in time series: diffusion entropy analysis, Physical Review E: statistical, nonlinear, and softmatter physics, 66, 036130, 2002.

12. Allegrini P., Benci V., Grigolini P., Hamilton P., Ignaccolo M., Menconi G., Palatella L., Raffaelli G., Scafetta N., Virgilio M., Yang J., Compression and diffusion: a joint approach to detect complexity, Chaos Solitons and Fractals, 15, 517-535, 2003.

13. Grigolini P., Palatella L., Raffaelli G., Asymmetric Anomalous Diffusion: an Efficient Way to Detect Memory in Time Series, Fractals, 9, 439-449, 2001. 